\title{
Duplicación de la vesícula biliar: Vesícula doble de tipo Y operada. Reporte de un caso*
}

\author{
Drs. JAVIER ELORZA D. ${ }^{1,2}$, SERGIO LÓPEZ S. ${ }^{3}$, BERNARDO ESPINOZA E. ${ }^{4}$, \\ FRANCISCO IZQUIERDO M. ${ }^{3}$, T M. VÍCTOR CARRIEL A. ${ }^{2}$ \\ Facultad de Medicina, Escuela de Medicina Campus San Felipe, Universidad de Valparaíso. \\ Unidad de Anatomía Patológica. \\ Servicio de Cirugía. \\ 4 Servicio de Radiología. \\ Hospital San Camilo, San Felipe, Chile.
}

\begin{abstract}
Gallbladder duplication. Report of one case

The duplication of the gallbladder is a rare malformation, seen in one every 3.000 necropsies. It is detected preoperatively by abdominal ultrasound. We report a 28 years old female consulting for abdominal pain. An abdominal ultrasound showed the presence of cholelithiasis and a complete septum in the gallbladder lumen. During surgery, a distended and inflamed gallbladder was found with a septum that divided it in two parts, and one biliary stone. There was also a double cystic duct. The pathological study confirmed the gallbladder duplication with inflammation.
\end{abstract}

Key words: Gallbladder, malformation, cholelithiasis.

\section{Resumen}

La patología de la vesícula biliar (VB) ocupa un lugar importante en nuestro país. Las anomalías congénitas más frecuentes incluyen alteraciones en los conductos biliares, arteria cística e infrecuentemente duplicaciones de la VB, con una incidencia de 1 en 3.000-4.000 autopsias. Esta anomalía congénita puede ser detectada preoperatoriamente por estudios imagenológicos, principalmente ecotomografía, siendo en el pasado un hallazgo quirúrgico. El diagnóstico diferencial de la duplicación vesicular incluye la vesícula con fondo plegado o gorro frigio, divertículos vesiculares, quistes de las vías biliares extra-hepáticas y la vesícula bilobulada o septada. Comunicamos el caso de una duplicación vesicular de tipo Y, en una mujer con diagnóstico ecotomográfico, la cual fue operada de forma clásica.

Palabras clave: Vesícula biliar, colecistectomía, duplicación vesicular.

\footnotetext{
Correspondencia: Dr. Javier Elorza D.

Avenida Miraflores 2085. San Felipe, Chile.

E-mail: elorzajavier@yahoo.com.ar
}

*Recibido el 22 de Agosto de 2008 y aceptado para publicación el 7 de Diciembre de 2008. 


\section{Introducción}

Comunicamos el caso de una mujer con diagnóstico ecotomográfico de duplicación vesicular operada de forma clásica.

\section{Caso Clínico}

Paciente mujer de 28 años quien consultó por dolor abdominal. El examen físico no mostró mayores alteraciones. Hemograma y perfil bioquímico normales. La ecotomografía muestra vesícula distendida, de paredes engrosadas y presencia de tabique completo en el espesor del lumen (Figura 1A). Se describe presencia de litiasis única de $1,8 \mathrm{~cm}$ en uno de los lúmenes identificados.

Se realizó cirugía abierta, por incisión subcostal transversal. Abierto el peritoneo se observa VB distendida, con paredes engrosadas, con signos de inflamación aguda. Se identifica un tabique central que separa la vesícula en dos (Figura 1B), con doble conducto cístico, que se fusionan confluyendo como uno solo en el hepático común, dando origen al colédoco. Una de las cavidades presenta litiasis única.

El estudio de anatomía patológica de la pieza operatoria muestra una duplicación vesicular, con signos inflamatorios en una de las vesículas y colesterolosis en la otra (Figura $1 \mathrm{~B}$ ). Los cortes histológicos evidencia la presencia de aspectos distintivos en ambas paredes vesiculares, como sig- nos de reagudización y colesterolosis. Se evidenció la presencia de mucosa a ambos lados del tabique, y dos capas fibromusculares independientes, lo que apoya el diagnóstico de una verdadera duplicación vesicular, y no una vesícula biliar septada.

\section{Discusión}

La duplicación de la VB es rara, pero descrita desde tiempos ancestrales, inicialmente en animales y luego en humanos desde la época del Emperador Augusto el año $31 \mathrm{aC}^{1}$. En 1674, Blasius describe el primer caso bien documentado en humanos. Estas no poseen síntomas específicos y las alteraciones patológicas no son más frecuentes en estos casos que en los pacientes con vesículas únicas $^{3,4}$. La formación de cálculos es la complicación más común, usualmente desarrollada en una vesícula $^{4,5}$, pero puede afectar ambas ${ }^{6}$. En 26 de 56 casos comunicados por Harlaftis ${ }^{4}$ se confirmó después de la cirugía que la litiasis afectaba sólo una vesícula. Las patologías más comunes de la VB han sido descritas en duplicaciones vesiculares, incluyendo la colecistitis aguda y empiema vesicular ${ }^{6}$, colecistitis crónica ${ }^{6}$, fístulas colecistocoledocianas ${ }^{7}$, torsión vesicular ${ }^{8}$, papilomas ${ }^{9}$, colesterolosis $^{10}$ y carcinomas vesiculares ${ }^{11}$. En algunas circunstancias raras se ha relacionado con lesiones más graves, que incluyen anomalías de los conductos biliares, arteria hepática, malformaciones cardíacas, riñones
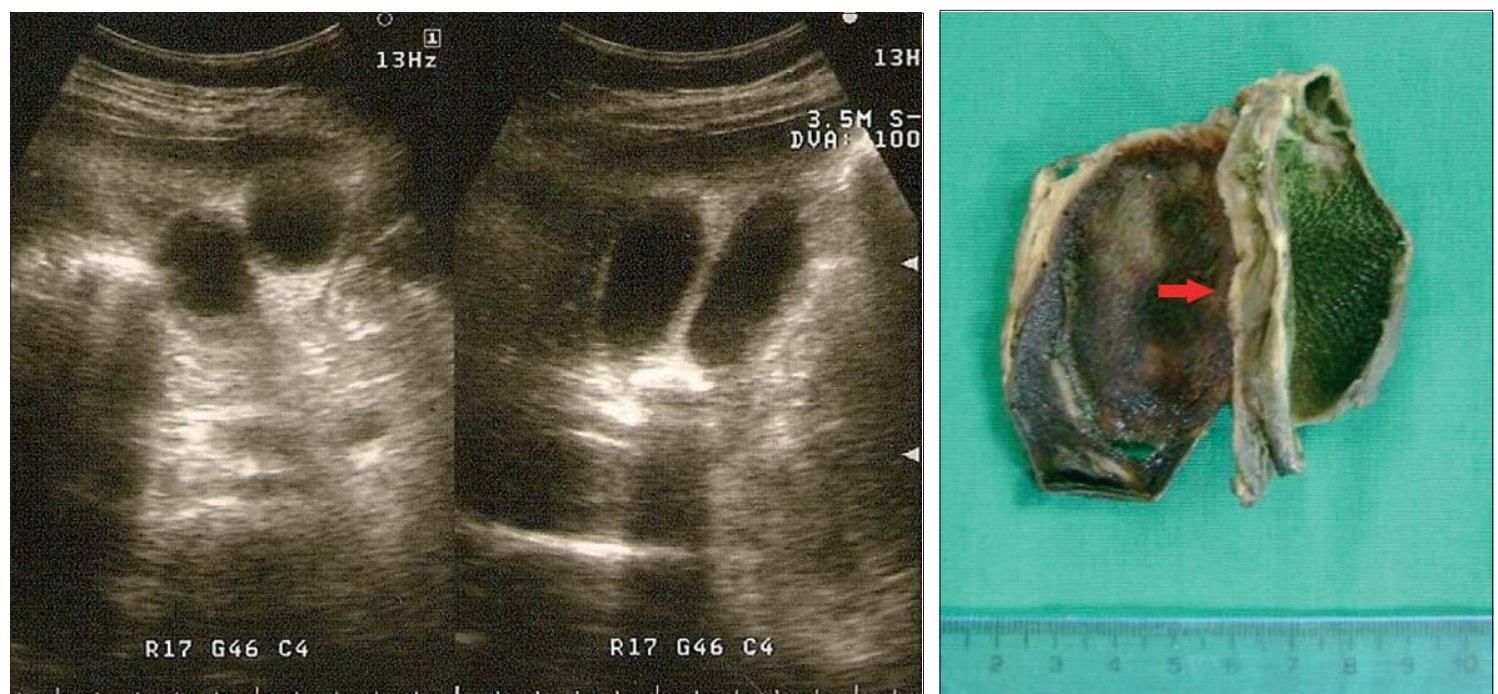

Figura 1. A) Ecotomografía abdominal que evidencia la presencia de dos lúmenes vesiculares y la sombra acústica de la

litiasis B) Pieza quirúrgica constituida por dos vesículas biliares, una con presencia de colesterolosis. La flecha señala la zona de fusión de ambas vesículas. 
poliquísticos y diversos defectos músculoesqueléticos ${ }^{4}$.

$\mathrm{Su}$ incidencia es de 1 en 3.000-4.000 autopsias comunicada por Boyden en una serie de $9.221 \mathrm{ca}-$ sos (dos en 9.221 autopsias) $^{1}$. En 1977, Harlaftis ${ }^{4}$ comunicó una serie de 207 duplicaciones de la vesícula biliar. Hess encontró un caso en $4.000(0,02 \%)$ pacientes operados ${ }^{12}$, mientras que Senecail diagnosticó con ecotomografía 3 casos en $1.823(0,16 \%)$ pacientes estudiados ${ }^{13}$.

Durante la cuarta semana de gestación la VB es una sólida excrescencia en continuidad con el divertículo hepático. Esta excrescencia crece rápidamente y entre la séptima a octava semana se canaliza y forma un saco. El pedículo se convierte en el conducto cístico, que conecta con el duodeno a través del colédoco ${ }^{14}$. Las verdaderas duplicaciones representan excrecencias independientes o secundarias del brote inicial en relación con el divertículo hepático ${ }^{15}$.

Las anomalías congénitas de la VB pueden clasificarse en alteraciones de localización, número o del desarrollo. Dentro de las alteraciones de localización la más habitual es la vesícula intra-hepática, que se localiza más frecuentemente en el lóbulo derecho. Las alteraciones en la rotación, durante el desarrollo embriológico del tubo digestivo o sus partes, producen localizaciones de la VB a la izquierda del ligamento falciforme ${ }^{14}$. Las alteraciones del número pueden traducirse en agenesia o duplicación de la VB. La agenesia es una condición ex- tremadamente rara observándose una incidencia en autopsias de $0,03 \%{ }^{1}$.

De acuerdo con la clasificación de Boyden (Figura 2), las verdaderas duplicaciones con dos diferentes conductos císticos, son de forma tipo Y o $\mathrm{H}$. La forma de tipo Y está constituida por dos VB adheridas ocupando la misma fosa, con dos conductos císticos separados que se fusionan para desembocar en el conducto hepático común y formar el colédoco ${ }^{1}$. Habitualmente en este caso una vesícula es más grande que la otra y ambas presentan arterias císticas independientes ${ }^{12,13}$. El caso que comunicamos corresponde a este tipo. La forma de tipo $\mathrm{H}$ o ductular está constituida por VB separadas con dos conductos císticos independientes que desembocan en el conducto hepático común separadamente para formar el conducto colédoco ${ }^{1}$. Ambas vesículas poseen arterias císticas independientes $^{12,13}$. Las VB triples también han sido descritas ${ }^{16}$.

Boyden también considera dentro de su clasificación las vesículas bilobuladas o septadas, la que corresponde a una VB divida por un tabique de tejido fibroconectivo o fibromuscular, sin presencia de mucosa en el tabique. En este caso sólo existe un conducto y una arteria cística. Esta condición constituye un importante diagnóstico diferencial para el radiólogo durante el examen ecotomográfico $^{17}$. Las anomalías del desarrollo incluyen la vesícula flotante, presente hasta en $10 \%$ de la población, vesícula con fondo plegado o gorro frigio, divertículos vesiculares, vesículas septada y pre-

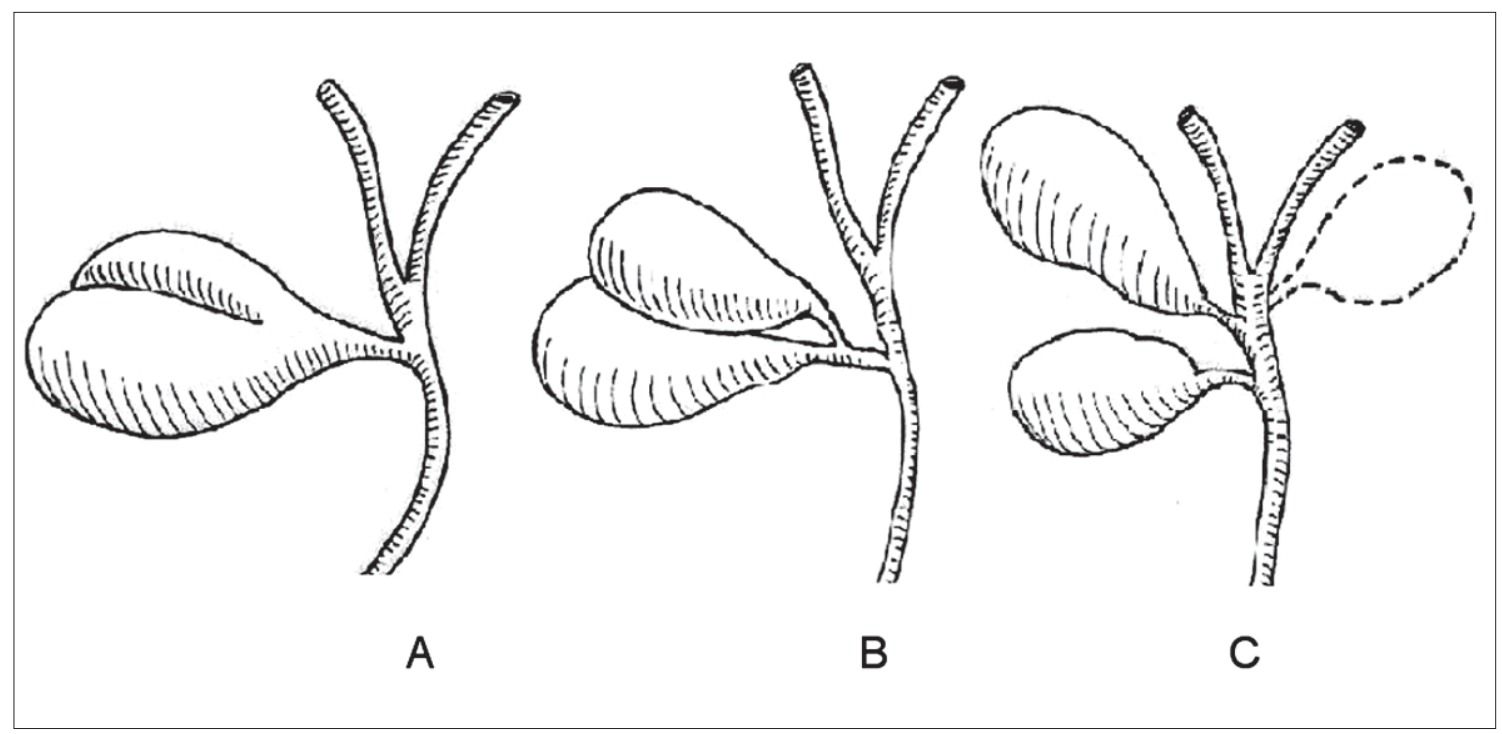

Figura 2. Tipos de duplicaciones vesiculares. A) Vesícula bilobulada (septada); B) duplicación vesicular de tipo Y; C) Duplicación vesicular de tipo H y sus posibles ubicaciones. 
sencia de tejido ectópicos en VB (como tejido hepático, gástrico, pancreático y tiroideo).

Para cirujanos, radiólogos y patólogos es importante conocer estas anomalías congénitas. Para el cirujano requiere especial atención para no causar lesiones iatrogénicas de la vía biliar, sobre todo teniendo presente la alta asociación de esta anomalía con alteraciones en la vía biliar ${ }^{12,13}$. El radiólogo debe tratar de distinguir la vesícula septada de las verdaderas duplicaciones vesiculares, en las cuales existen conductos císticos independientes, desembocando juntos o separadamente en el hepático común ${ }^{17}$.

La descripción del conducto cístico y su relación con la vía biliar, durante la ecotomografía o por medio de estudios complementarios como la colangio-resonancia, agregan datos importantes para definir la forma de abordaje quirúrgico. Los anatomopatólogos durante el examen macroscópico deben prestar atención al hecho que frecuentemente una de las dos vesículas es más pequeña que la otra, lo que toma mucha importancia en el examen macroscópico de VB dobles fusionadas como la de tipo $\mathrm{Y}$, casos en los cuales puede ser inadvertida la duplicación si una de las dos vesículas es demasiado pequeña ${ }^{13}$.

Se recomienda la remoción quirúrgica de ambas vesículas durante la cirugía, pues se ha reportado reintervenciones quirúrgicas por complicaciones en pacientes con duplicaciones vesiculares no removidas totalmente durante la primera cirugía. La opción laparoscópica es una alternativa menos invasiva de solución quirúrgica, sobre todo en los casos en los cuales la vía biliar ha sido adecuadamente estudiada ${ }^{18}$.

Nos ha parecido interesante comunicar este caso pues no encontramos otras publicaciones nacionales que muestren una duplicación vesicular de tipo Y diagnosticada con ecotomografía y operada.

\section{Referencias}

1. Boyden EA. The accesory gall-bladder-an embryological and comparative study of aberrant biliary vesicles occurring in man and the domestic mammals. Am J Anat 1926; 38: 177-231.

2. Van Wiechen PJ. Gallbladder duplication. J Belge Radiol-BTR 1994; 77: 227.
3. Harlaftis N, Gray SW, Olafson RP, Skandalakis JE. Three cases of unsuspected double gallbladder. Am Surg 1976; 3: 178-180.

4. Harlaftis N, Gray SW, Olafson RP, Skandalakis JE. Multiple gallbladders. Surg Gynecol Obst 1977; 145: 928-934.

5. Díaz MJ, Fowler W, Hnatow BJ. Congenital gallbladder duplicaton: preoperative diagnosis by ultrasonography. Gastrointest Radiol 1991; 16: 198-200.

6. Cueto García J, Webwr A, Berry FS, Tatz BT. Double gallbladder treated successfully by laparoscopy. J Laparoendosc Surg 1993; 3: 153-155.

7. Ritchie AWS, Crucioli V. Double gallbladder with cholecysto-colic fistula: a case report. Br J Surg 1980; 67: $145-146$.

8. Recht W. Torsion of a double gallbladder: a report of a case and rewiew of literature. Br J Surg 1951; 39: 342-344.

9. Roeder WJ, Mersheimer WL, Kazarin KK. Triplication of the gallbladder with cholecystitis, cholelithiasis and papillary adenocarcinoma. Am J Surg 1971; 121: 746748.

10. Gautman A, Kala S, Kumar M, Sharma CI. Double gallbladder with two disease processes. Indian $\mathrm{J}$ Gastroenterol 1999; 18: 179-180.

11. Raymond SW, Thrift CB. Carcinoma of a duplicated gallbladder. III Med J 1956; 110: 239-240.

12. Hess W. Enfermedades de las vías biliares y del páncreas. Barcelona: Editorial Científico-médica; 1963: 3-37.

13. Senecail B, Textier F. Anatomic variability and congenital anomalies of gallbladder: ultrasonographic study of 1823 patients. Morphologie 2000; 84: 35-39.

14. Sadler T. Embriología médica de Langman. Editorial Panamericana octava edicion.

15. Van Steenberger W, Krekelberg F, Ectors N, Ponette E, Yap SH. Double gallbladder documented by endoscopic retrograde cholangiography. J Belge Radiol-BTR 1993; 76: 243-244.

16. Foster DR. Triplle gallbladder. Br J Radiol 1981; 54: 817-818.

17. Mohammad A, Gerbail T, Glowniak V. Identification and differentiation of congenital gallbladder abnormality by quantitative technetium-99m IDA cholescintigraphy. The Journal of Nuclear Medicine 1992; 33: 431-434.

18. Mackie DB. Diagnosis and treatment of duplication of the gallbladder. Postgrad Med 1966; 42: 213-216. 